\title{
C. ANTOINE 1,2, 曲 \\ Matter wave beam splitters in gravito-inertial and trapping potentials: generalized ttt scheme for atom interferometry
}

${ }^{1}$ ERGA, LERMA, CNRS-Observatoire de Paris, Université P. et M. Curie, 75005 Paris, France

${ }^{2}$ Group of Gravitation and Experiments with Cold Atoms, TIFR, 400005 Mumbai, India

\section{Published online: 23 August 2006 • ㄷ Springer-Verlag 2006}

Several intermediate expressions of the paragraph " 6.1 First-order resolution' have to be corrected. These errors in formulas occurred during the preparation of the manuscript. There is no consequence for the other paragraphs, since the results presented in this article were all obtained using the correct expressions.

In paragraph '6.1 First-order resolution', one should read: $\ldots$

To the first order in $\boldsymbol{k} \cdot \boldsymbol{g} \tau^{2}$, the solution of (11) is obtained readily as

$S(\tau)=\left(\begin{array}{cc}\cos (\Omega \tau)+\mathrm{i} \frac{y}{\sqrt{1+y^{2}}} \sin (\Omega \tau) & \mathrm{i} \frac{1}{\sqrt{1+y^{2}}} \sin (\Omega \tau)+a \\ \mathrm{i} \frac{1}{\sqrt{1+y^{2}}} \sin (\Omega \tau)-a & \cos (\Omega \tau)-\mathrm{i} \frac{y}{\sqrt{1+y^{2}}} \sin (\Omega \tau)\end{array}\right)$,

where $t_{1}$ has been chosen equal to the central time of interaction, $t_{1}=\left(t+t_{0}\right) / 2$, and where the quantity $a$ is defined as

$a:=\boldsymbol{k} \cdot \boldsymbol{g} \tau^{2} \frac{1}{\sqrt{1+y^{2}}} \frac{1}{4 \Omega \tau}\left(\frac{\sin (\Omega \tau)}{\Omega \tau}-\cos (\Omega \tau)\right)$

Once made independent of $\boldsymbol{p}, S(\tau)$ is a pure numerical matrix whose elements can be expressed in terms of phase and amplitude (to the first order in $\left.\boldsymbol{k} \cdot \boldsymbol{g} \tau^{2}\right)$ :

$S(\tau)=\left(\begin{array}{ll}\varrho_{b b} \mathrm{e}^{\mathrm{i} \varphi_{b b}} & \varrho_{b a} \mathrm{e}^{\mathrm{i} \varphi_{b a}} \\ \varrho_{a b} \mathrm{e}^{\mathrm{i} \varphi_{a b}} & \varrho_{a a} \mathrm{e}^{\mathrm{i} \varphi_{a a}}\end{array}\right)$,

with $\varphi_{b b}=\tan ^{-1}\left[\frac{y_{1}}{\sqrt{1+y_{1}^{2}}} \tan \left(\Omega_{1} \tau\right)\right]$

$\varphi_{a a}=-\varphi_{b b}$,

$\varphi_{b a}=\frac{\pi}{2}-\boldsymbol{k} \cdot \boldsymbol{g} \tau^{2} \frac{1}{4 \Omega_{1} \tau}\left(\frac{1}{\Omega_{1} \tau}-\cot \left(\Omega_{1} \tau\right)\right)$,

$\varphi_{a b}=\pi-\varphi_{b a}$

and

$\varrho_{a a}=\varrho_{b b}=\sqrt{\cos ^{2}\left(\Omega_{1} \tau\right)+\frac{y_{1}^{2}}{1+y_{1}^{2}} \sin ^{2}\left(\Omega_{1} \tau\right),}$
$\varrho_{a b}=\varrho_{b a}=\frac{1}{\sqrt{1+y_{1}^{2}}}\left|\sin \left(\Omega_{1} \tau\right)\right|$

If the second condition of the Borrmann effect is also fulfilled (i.e. $\left.\left|y_{1}\right| \ll 1\right)$, the transfer matrices corresponding to the $\pi / 2$ and $\pi$ beam splitters become simply

$S_{\pi / 2}(\tau)$

$=\frac{1}{\sqrt{2}}\left(\begin{array}{cc}\exp \left[\mathrm{i} y_{1}\right] & \mathrm{i} \exp \left[-\mathrm{i} \boldsymbol{k} \cdot \boldsymbol{g} \tau^{2} \frac{4}{\pi^{2}}\left(1-\frac{\pi}{4}\right)\right] \\ \mathrm{exp}\left[-\mathrm{i} y_{1}\right]\end{array}\right)$,

$S_{\pi}\left(\tau_{\pi}=2 \tau_{\pi / 2}=2 \tau\right)=\left(\begin{array}{cc}\mathrm{i} y_{1} & \mathrm{i} \exp \left[-\mathrm{i} \boldsymbol{k} \cdot \boldsymbol{g} \tau^{2} \frac{4}{\pi^{2}}\right] \\ \mathrm{i} \exp \left[+\mathrm{i} \boldsymbol{k} \cdot \boldsymbol{g} \tau^{2} \frac{4}{\pi^{2}}\right] & \mathrm{i} y_{1}\end{array}\right)$. 